\title{
A CATALOG OF DUSTY ELLIPTICAL GALAXIES
}

\author{
KATE EBNETER ${ }^{\text {a) }}$ \\ Astronomy Department, University of California at Berkeley, Berkeley, California 94720 \\ and Department of Astronomy, FM-20, University of Washington, Seattle, Washington 98195 \\ BRUCE BALICK ${ }^{a), b), c) ~}$ \\ Department of Astronomy, FM-20, University of Washington, Seattle, Washington 98195 \\ and Sterrewacht Leiden, Leiden, The Netherlands \\ Received 18 September 1984; revised 6 November 1984
}

\begin{abstract}
A catalogue of early-type galaxies containing anomalous amounts and/or distributions of dust is compiled from the literature. The biases inherent in the catalogue are discussed. New CCD observations of selected dusty elliptical galaxies are presented that help to clarify the configuration of the dust. The morphological types, radio properties, radio-source orientations, neutral-hydrogen contents, and kinematics of the catalogue galaxies are reviewed, and the possible correlations between the presence and distribution of dust, on the one hand, are compared to other attributes of these galaxies, on the other hand.
\end{abstract}

\section{INTRODUCTION}

Attention has recently been drawn to a class of apparently elliptical galaxies which contain various quantities of dust, usually (but not always) in a lane along an optical axis of the galaxy (Bertola and Galletta 1978; Kotanyi and Ekers 1979; Bertola 1981; Hawarden et al. 1981; Kotanyi 1981; Tohline, Simonson, and Caldwell 1982). The archetypal "dusty elliptical" is the well-known radio galaxy NGC 5128 (Centaurus A; for a recent review, see Ebneter and Balick 1983), which appears to be a normal elliptical galaxy bisected by a thick, warped dust lane which lies along the galaxy's minor axis. The presence of dust in early-type systems which are normally found to be free of dust and gas has led to a great deal of interest in this class of galaxies. The fact that many such galaxies are found to be radio sources has also promoted the suggestion that the gas associated with the dust in these galaxies provides a source of fuel for the active nucleus (see Gunn 1979). Hence, the dusty ellipticals may also be important for our understanding of the origins of activity in elliptical galaxies. Furthermore, the dust lanes in some of these galaxies provide information about the dynamics and the true, three-dimensional figures of the galaxies.

The study of dusty ellipticals has been somewhat hampered by the lack of any single, large list of early-type systems containing dust, although many such galaxies have been identified in the literature. This paper contains a cata$\log$ of some one hundred dusty ellipticals and some related objects, culled from the literature (the literature search was completed in 1984 April). A number of examples are illustrated. Section II contains the catalog and a description of the various types of dusty ellipticals; a morphological classification system is proposed. Observations of a sample of dusty ellipticals are discussed in Sec. III. In Sec. IV, the known properties of the sample are discussed; the hypoth-

\footnotetext{
a) Visiting Astronomer at Kitt Peak National Observatory (KPNO) of the National Optical Astronomy Obervatories (NOAO). The NOAO is operated by Associated Universities for Research in Astronomy under contract to the National Science Foundation.

${ }^{b)}$ Visiting Astronomer at Cerro Tololo Interamerican Observatory of the NOAO.

${ }^{c)}$ On sabbatical leave at Sterrewacht Leiden 1983-1984.
}

eses which have been proposed to explain these galaxies and their relationship to other types of peculiar galaxies are discussed in Sec. V.

\section{THE CATALOG}

The present catalog (Table I) was compiled primarily from previously published lists of dusty early-type galaxies and from published reports of dust in individual objects. (Somewhat surprisingly, most of these lists do not overlap very much). In particular, all objects from the list of Hawarden et al. (1981), and most of the galaxies observed by Longmore and Sharples (1982), have been included. (A few of the latter have been excluded as being more probably of the M82 type; another, $0547-245$, was excluded because it could not be positively identified.) All elliptical galaxies identified in published papers as containing dust have been included. In addition, The Hubble Atlas of Galaxies (Sandage 1961) and the Atlas of Peculiar Galaxies (Arp 1966) were searched for candidates. Some galaxies from the latter source are more or less disturbed in appearance, but were included on the grounds that they resemble ellipticals more than anything else. Finally, all galaxies classified as Ep by Morgan $(1958,1959)$ were investigated; most of these were already known from other sources. All galaxies which were expected to show largescale dust structures were then inspected on either the Palomar Sky Survey prints or the ESO(B) Sky Survey film copies (occasionally both) to confirm the presence of dust and the dust-lane morphology.

Generally, borderline cases have been included. Galaxies which seemed to be of the M82 class of irregular or amorphous galaxies were excluded (see Sec. IV); these galaxies tend to be highly flattened. The catalog does, however, differ from that of Hawarden et al. (1981) in that the present list does not discriminate against galaxies in which the dust is not in a well-defined lane. Four severely distorted systems were excluded from the main catalog (see Table II) but are discussed in the present paper because they may be important for understanding the origins of the dusty ellipticals.

While every attempt has been made to include all known dusty early-type galaxies, the catalog is in no sense complete, for a number of reasons. There are several selection effects to which this catalog is particularly susceptible. More of the galaxies in the southern hemisphere were discovered for 
TABLE I. Elliptical galaxies with dust.

\begin{tabular}{|c|c|c|c|c|c|c|c|c|c|c|c|}
\hline $\begin{array}{l}\text { (1) } \\
\text { ID }\end{array}$ & $\begin{array}{l}(2) \\
R A(1950)\end{array}$ & $\begin{array}{l}\text { (3) } \\
\mathrm{DEC}(1950)\end{array}$ & $\begin{array}{l}(4) \\
\text { TYPE }\end{array}$ & $\begin{array}{l}(5) \\
B\end{array}$ & $\begin{array}{l}\text { (6) } \\
\mathrm{v}\end{array}$ & $\begin{array}{l}(7) \\
\mathrm{HI}\end{array}$ & $\begin{array}{l}(8) \\
S(1.4 \mathrm{GHz}) \\
\end{array}$ & $\begin{array}{l}\text { ( } 9 \text { ) } \\
\text { REFERENCES }\end{array}$ & $\begin{array}{l}(10) \\
\text { NOTES }\end{array}$ & $\begin{array}{l}(11) \\
\text { PHOTO }\end{array}$ & $\begin{array}{l}(12) \\
\text { COMENTS } \\
\end{array}$ \\
\hline $\begin{array}{l}0036+481 \\
0037\end{array}$ & $\begin{array}{llll}00 & 36 & 11 \\
00 & 37 & 38\end{array}$ & 480342 & $D$ & 10.1 & 4 & $\mathrm{em}$ & $<0.10$ & $20,55,60$ & 1,8 & 60 & NGC 185; Local Group \\
\hline 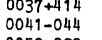 & $\begin{array}{lll}00 & 3738 \\
00 & 41 & 01\end{array}$ & 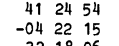 & $\begin{array}{l}\mathrm{D} \\
\mathrm{P}, \mathrm{BE}\end{array}$ & 8.85 & 1 & $\mathrm{em}$ & $<0.05$ & $\begin{array}{l}20,55,60 \\
1,2,36\end{array}$ & $\frac{1,8}{10}$ & $\begin{array}{l}60 \\
40\end{array}$ & $\begin{array}{l}\text { NoC 205; Local Group } \\
\text { IC 1575=Arp 231 }\end{array}$ \\
\hline $\begin{array}{l}0052-323 \\
0055+300\end{array}$ & 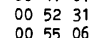 & $\begin{array}{rrr}-32 & 18 & 06 \\
30 & 04 & 54\end{array}$ & $\begin{array}{l}0 \\
D\end{array}$ & 12.5 & & & & $\begin{array}{l}1,2,36 \\
3,65,68\end{array}$ & & & $\begin{array}{l}\text { IC } 1575=\operatorname{Arp} 231 \\
\text { MCG }-5-3-13\end{array}$ \\
\hline $\begin{array}{l}0 \\
0104+321\end{array}$ & 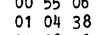 & $\begin{array}{l}30 \quad 0454 \\
320842\end{array}$ & $\begin{array}{l}D \\
0\end{array}$ & $\begin{array}{l}12.5 \\
12.9\end{array}$ & $\begin{array}{l}518 \\
5095\end{array}$ & $\begin{array}{l}\text { ab } \\
\text { nd }\end{array}$ & $\begin{array}{l}1.8 \\
4.7\end{array}$ & $\begin{array}{l}3,65,68 \\
3,65\end{array}$ & $\begin{array}{l}1,3,4 \\
1,3,4\end{array}$ & & $\begin{array}{l}\text { NGC } 315 ; \text { DW } 0055+26 \\
\text { NGC 383; } 3 C 31=\text { PRS } 0104+32\end{array}$ \\
\hline $\begin{array}{l}0106+355 \\
0123-019\end{array}$ & $\begin{array}{llll}01 & 06 & 36 \\
01 & 23 & 52\end{array}$ & $\begin{array}{r}3527 \\
-015354\end{array}$ & $\begin{array}{l}\mathrm{P} \\
\mathrm{O}\end{array}$ & & $\begin{array}{r}178 \\
5715\end{array}$ & $\mathrm{em}$ & $<0.05$ & $\begin{array}{l}3,03 \\
20,60 \\
2\end{array}$ & & 60 & $\begin{array}{l}\text { NGC 383; 3C 31=PRS } 0104+32 \\
\text { NGC 404; Local Group? }\end{array}$ \\
\hline $\begin{array}{l}0131-367 \\
013-246\end{array}$ & 013144 & $\begin{array}{l}-0153 \\
-3644 \\
-24\end{array}$ & $0, \mathrm{BB}$ & 14.1 & $\begin{array}{l}5715 \\
9049\end{array}$ & & 10.9 & $\begin{array}{l}2 \\
5,6\end{array}$ & $\begin{array}{l}5 \\
3,4\end{array}$ & 6 & $\begin{array}{l}\text { IC } 1703 \\
\text { NGC } 612 ; \text { PRS 0131-36 }\end{array}$ \\
\hline $\begin{array}{l}0132-246 \\
0141+374\end{array}$ & $\begin{array}{l}013236 \\
014154\end{array}$ & $\begin{array}{rl}-24 & 41 \\
3726\end{array}$ & $\begin{array}{l}0 ? \\
\text { SPP? } \\
S W H\end{array}$ & 14.7 & 5885 & & & $\begin{array}{l}36 \\
2,57\end{array}$ & 10 & & NGC 662 \\
\hline $\begin{array}{l}0147-269 \\
0149+359\end{array}$ & $\begin{array}{l}014706 \\
014954\end{array}$ & $\begin{array}{l}-265957 \\
3555\end{array}$ & $\begin{array}{l}\text { S, W } \\
\text { P/S }\end{array}$ & 14.8 & 5023 & nd & 0.17 & $8,9,65$ & $\begin{array}{l}5 \\
5,6\end{array}$ & & 14c0 \\
\hline $\begin{array}{l}0151-498 \\
0206+355\end{array}$ & $\begin{array}{ll}01 & 5116 \\
02 & 06 \\
0 & 39\end{array}$ & $\begin{aligned}-494818 \\
-353\end{aligned}$ & $\mathrm{P}$ & 14.1 & $\begin{array}{r}6170 \\
\end{array}$ & & & $\begin{array}{l}8,9,65 \\
1,2,7\end{array}$ & & $\begin{array}{l}4,7 \\
1,7\end{array}$ & NGC 708; B2 $0149+35 B$ \\
\hline $0206+355$ & 020639 & $35 \quad 3341$ & $\mathrm{P}$ & 14.7 & 11075 & & 2.08 & 8,9 & 3 & & $\begin{aligned} \text { OGC } & 01651 ; \text { B2 } 0206+35 \\
= & =4 C 35.05\end{aligned}$ \\
\hline $\begin{array}{l}0219-345 \\
0226-109\end{array}$ & $\begin{array}{lll}02 & 19 & 10 \\
02 & 26 & 54\end{array}$ & $\begin{array}{l}-343234 \\
-1058\end{array}$ & $\begin{array}{l}s \\
0\end{array}$ & & & & & 40 & $\begin{array}{l}5 \\
9\end{array}$ & $\begin{array}{l}1 \\
40\end{array}$ & $\begin{array}{l}=4 \mathrm{C35} .05 \\
\text { MaC } 943=\operatorname{Arp} 309\end{array}$ \\
\hline $\begin{array}{l}0238-085 \\
0301-158\end{array}$ & $\begin{array}{lll}02 & 38 & 37 \\
03 & 01 & 19\end{array}$ & $\begin{array}{l}-0828 \quad 06 \\
-1548 \quad 36\end{array}$ & $\stackrel{P}{\mathrm{v}}$ & 11.5 & $\begin{array}{l}1434 \\
2645\end{array}$ & $e m+a b$ & 1.20 & 37 & $1,4,5$ & 37 & 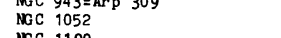 \\
\hline $0316-193$ & 031658 & $\begin{array}{l}-191648 \\
-19\end{array}$ & P? & 12.6 & 1471 & & $<0.05$ & $\begin{array}{l}42, \\
2\end{array}$ & & & $\begin{array}{l}\text { NGC } 1199 \\
\text { NGC }\end{array}$ \\
\hline $\begin{array}{l}0320-374 \\
0326-312\end{array}$ & 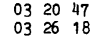 & 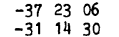 & l $\begin{array}{l}\mathrm{D}, \mathrm{P} \\
\text { U(D\}) }\end{array}$ & $\begin{array}{r}91.7 \\
11.2\end{array}$ & $\begin{array}{l}1632 \\
1088\end{array}$ & nd & 115 & 10,36 & $3,4,5,9,10$ & 10 & NGC 316 ; Fornax A \\
\hline $\begin{array}{l}0413-562 \\
0418-583\end{array}$ & $\begin{array}{llll}0 & 4 & 13 & 32 \\
04 & 18 & 03\end{array}$ & 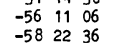 & $\begin{array}{l}0 \\
\mathrm{U}\end{array}$ & 12.5 & 935 & & & $\begin{array}{l}49 \\
2 \\
2\end{array}$ & $\begin{array}{l}10 \\
5\end{array}$ & & $\begin{array}{l}\text { NGC } 1344 \\
\text { NGC } 1546\end{array}$ \\
\hline $\begin{array}{l}0526-638 \\
0532-527\end{array}$ & $\begin{array}{ll}0526 & 29 \\
05 & 3205\end{array}$ & $\begin{array}{rl}-56348 & 30 \\
-524033 & \end{array}$ & P & 11.8 & 653 & & 0.046 & $\begin{array}{l}2 \\
1,2,11,12,48\end{array}$ & & 11,12 & NGC 1947 \\
\hline 作 $0544-168$ & 054435 & $\begin{array}{l}-524030 \\
-1648\end{array}$ & $\begin{array}{l}0 \\
0\end{array}$ & & & & & ${ }_{2}^{2}$ & 5 & & NGC 2076 \\
\hline 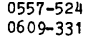 & 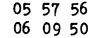 & $\begin{array}{lll}-52 & 27 & 06 \\
-33 & 07 & 01\end{array}$ & $\begin{array}{l}0 \\
\mathrm{P}\end{array}$ & & & & & 1 & & & \\
\hline $\begin{array}{l}0632-629 \\
0632-346\end{array}$ & $\begin{array}{l}063252 \\
06\end{array}$ & $\begin{array}{l}-35010 \\
-625719 \\
-34\end{array}$ & P & & & & & $\begin{array}{l}1 \\
1,36(3)\end{array}$ & $5,10(8)$ & & \\
\hline $\begin{array}{l}0639-346 \\
0641-412\end{array}$ & $\begin{array}{l}063951 \\
064152\end{array}$ & 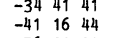 & $\begin{array}{l}0 \\
0\end{array}$ & & & & & $\stackrel{1}{1}$ & & & \\
\hline $\begin{array}{l}\begin{array}{l}0745+560 \\
0808+558\end{array} \\
080\end{array}$ & $\begin{array}{l}074547 \\
080856\end{array}$ & $\begin{array}{l}560200 \\
554916\end{array}$ & $\begin{array}{l}\text { D } \\
I\end{array}$ & & $\begin{array}{r}10688 \\
3625\end{array}$ & & $\begin{array}{l}3.8 \\
<0.05\end{array}$ & $\begin{array}{l}50 \\
1\end{array}$ & & 50 & $\begin{array}{l}\text { MCG 9-13-57; DA } 240=4056.16 \\
\text { NGC 2534 }\end{array}$ \\
\hline $\begin{array}{l}0828+411 \\
0907+602\end{array}$ & $\begin{array}{l}082808 \\
0907\end{array}$ & $\begin{array}{ll}41 & 0648 \\
60 & 1430\end{array}$ & $\underset{D}{I}$ & 10.9 & 1502 & $\mathrm{em}$ & $<0.05$ & $1,2,51$ & 4 & & \\
\hline 0929-165 & 092915 & $\begin{array}{rl}-16 & 14300 \\
-16 & 00\end{array}$ & 0 & $\begin{array}{l}13.9 \\
13.0\end{array}$ & $\begin{array}{l}150 c \\
1811\end{array}$ & en & $<0.05$ & $\begin{array}{l}4,39,43 \\
2,39\end{array}$ & 4 & & $\begin{array}{l}\text { NGC } \\
\text { NGC } \\
2\end{array}$ \\
\hline $\begin{array}{l}0931+104 \\
0 \\
0.40+322\end{array}$ & 093105 & $\begin{array}{ll}10 & 2254 \\
32 & \end{array}$ & D & 12.6 & 3026 & $e m+a b$ & 0.18 & & 4,5 & $\begin{array}{l}39 \\
40\end{array}-10$ & NGC $2919=\operatorname{Arp} 232$ \\
\hline $\begin{array}{l}0940+322 \\
0958-314\end{array}$ & $\begin{array}{l}094013 \\
095828\end{array}$ & $\begin{array}{rl}3210 & 10 \\
-31 & 2524\end{array}$ & $\begin{array}{l}S \\
v\end{array}$ & 12.8 & 1564 & & 0.10 & $\begin{array}{l}2 \\
56\end{array}$ & 5,9 & & $\begin{array}{l}\text { NGC } 2968 ; \text { B2 } 0940+32 \mathrm{~B} \\
\text { NGC } 3100\end{array}$ \\
\hline $\begin{array}{l}1000-314 \\
1029-459\end{array}$ & 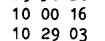 & 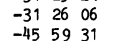 & ${ }_{0}^{\mathrm{P}(\mathrm{M} ?)}$ & 13.6 & 2597 & & 0.032 & $\begin{array}{l}30,56 \\
14,56\end{array}$ & 5 & 56 & NGC 3108 \\
\hline $1029+544$ & 102922 & $\begin{array}{rl}-4 & 523921 \\
543924\end{array}$ & $\mathrm{P}, \mathrm{D}$ & 13.0 & 1693 & & 0.032 & $\begin{array}{l}1,46,47,51 \\
40,57\end{array}$ & 3,5 & $\begin{array}{l}1 \\
40\end{array}$ & Arp 233 \\
\hline $\begin{array}{l}1033-321 \\
1034-273 \mathrm{~A}\end{array}$ & $\begin{array}{lll}100 & 33 & 27 \\
10 & 34 & 14\end{array}$ & $\begin{array}{lllll}-32 & 06 & 23 \\
-27 & 15 & 36\end{array}$ & $\begin{array}{l}0 \\
D\end{array}$ & 12.9 & 3818 & & & 14 & $\begin{array}{l}9 \\
5,\end{array}$ & 44 & $\begin{array}{l}\text { NGC } 3302 \\
\text { NGC } 3309\end{array}$ \\
\hline $\begin{array}{l}1034-2738 \\
1040+776\end{array}$ & 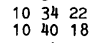 & $\begin{array}{l}-271606 \\
7737\end{array}$ & $\begin{array}{l}\mathrm{D} \\
\mathrm{O} / \mathrm{s}, \mathrm{W}\end{array}$ & 12.5 & 3354 & & & $\begin{array}{l}15,44,57 \\
40\end{array}$ & $\begin{array}{l}3,5,9 \\
1,5,9\end{array}$ & $\begin{array}{l}44 \\
40\end{array}$ & $\begin{array}{l}\text { NaC } 3311 \\
\text { Arp } 156\end{array}$ \\
\hline $\begin{array}{l}1104-392 \\
1104-102\end{array}$ & $\begin{array}{l}110450 \\
11104\end{array}$ & $\begin{array}{ll}-391150 \\
-3911506\end{array}$ & $0^{0}$ & & & & & 1 & & & $\begin{array}{l}\text { Arp } 156 \\
\text { NaC } 3523\end{array}$ \\
\hline $\begin{array}{l}\begin{array}{l}11044-192 \\
1118-291\end{array} \\
110\end{array}$ & 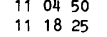 & 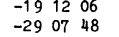 & $\begin{array}{l}0 \\
0, \mathrm{w}\end{array}$ & & & & 0.0522 & 2 & $\begin{array}{l}5,9,11 \\
11\end{array}$ & $:$ & $\begin{array}{l}\text { NGC } 3528 \\
\text { MCG }-5-27-10\end{array}$ \\
\hline $1120+541$ & 112049 & 54 o7 06 & $\mathrm{P}, \mathrm{D}$ & & 2910 & & $<0.10$ & 49 & & 40 & NGC $3656=$ Arp 155 \\
\hline $\begin{array}{l}1122+390 \\
11177+80\end{array}$ & 112201 & 390211 & 0 & 11.7 & 2012 & nd & 0.11 & $\begin{array}{l}2,5,8,16 \\
6465\end{array}$ & $3,4,5,11$ & 16 & $\operatorname{Mgc} 3665 ;$ B2 $1122+39$ \\
\hline $\begin{array}{l}1137+180 \\
1148-285\end{array}$ & $\begin{array}{l}113741 \\
114830\end{array}$ & $\begin{array}{rl}18 & 0024 \\
-28 & 3142\end{array}$ & $\begin{array}{c}M \\
D\end{array}$ & $\begin{array}{l}13.0 \\
11.1\end{array}$ & $\begin{array}{l}3174 \\
1546\end{array}$ & $\begin{array}{l}\mathrm{em}+\mathrm{ab} \\
\mathrm{nd}\end{array}$ & $\begin{array}{l}0.096 \\
<0.15\end{array}$ & $\begin{array}{l}64,65 \\
36,49\end{array}$ & ${ }_{1,5,10}^{9}$ & 36 & $\begin{array}{l}\text { NGC } 3801 ; 4 C 17.52 \\
\text { NGC 3923 }\end{array}$ \\
\hline $\begin{array}{l}1153+433 \\
1205+655\end{array}$ & 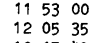 & $\begin{array}{l}4319 \\
652718\end{array}$ & $\begin{array}{l}\circ \\
\mathrm{s}\end{array}$ & 10.7 & 1482 & nd & 0.06 & $\begin{array}{l}2 \\
52\end{array}$ & & & $\begin{array}{l}\text { UGC } 06901 \\
\text { NGC } 4125\end{array}$ \\
\hline $\begin{array}{l}1217+296 \\
1222+077\end{array}$ & $\begin{array}{l}121742 \\
122223\end{array}$ & $\begin{array}{l}2934 \\
0743313\end{array}$ & $D_{0}^{D}$ & 14.1 & $\begin{array}{l}651 \\
685\end{array}$ & $\begin{array}{c}\text { em } \\
\text { em }\end{array}$ & 0.50 & 53,58 & 11 & $\because$ & NAC 4278; B2 $1217+29$ \\
\hline $\begin{array}{l}1222++477 \\
1222+132 \\
1235+337\end{array}$ & 122231 & $\begin{array}{ll}13 & 0948 \\
3 & 48\end{array}$ & ${ }_{\mathrm{P}}^{\mathrm{P}}$ & 10.3 & 854 & nd & 0.6 & $\begin{array}{l}1,2,41,41,65 \\
1,2,19,41,65\end{array}$ & $3,4,5$ & 4,19 & $\begin{array}{l}\text { NGC } 4370 \\
\text { NGC } 4374=\text { M } 84 ; 3 C 272 \text { (Virgo) }\end{array}$ \\
\hline $\begin{array}{l}1235+337 \\
1246-410\end{array}$ & $\begin{array}{lll}12 & 35 & 35 \\
12 & 46 & 03\end{array}$ & $\begin{array}{rlll}33 & 44 & 18 \\
-41 & 02 & 18\end{array}$ & $\begin{array}{l}p_{0} \\
0\end{array}$ & 11.7 & 2690 & & 3.93 & 2 & & 54 & $\begin{array}{l}\text { NGC 4583; } \\
\text { NGC 469; PRS } 1246-41\end{array}$ \\
\hline $\begin{array}{l}1240-009 \\
107-467\end{array}$ & 124948 & $\begin{array}{l}-005552 \\
-46\end{array}$ & S, D & 10.8 & $\begin{array}{l}1137 \\
1620\end{array}$ & & & & si & 60 & NGC 4753 \\
\hline $\begin{array}{l}13010-301 \\
1310-302\end{array}$ & $\begin{array}{lll}13 & 10 & 10 \\
13 & 10 & 10\end{array}$ & $\begin{array}{lll}-46 & 4329 \\
-30 & 10 & 10 \\
-30 & \end{array}$ & $\begin{array}{l}S(D ?) \\
0\end{array}$ & 13.0 & 1620 & & 0.0068 & $\begin{array}{l}1,46,47 \\
1,2,51\end{array}$ & 11 & & \\
\hline $\begin{array}{l}1310-193 \\
1320-324\end{array}$ & $\begin{array}{lll}13 & 10 & 20 \\
13 & 20 & 08\end{array}$ & $\begin{array}{lll}-19 & 15 & 12 \\
-32 & 28 & 21\end{array}$ & ${ }_{\mathrm{I}}^{\mathrm{U}(\mathrm{D} ?)}$ & 11.8 & 2729 & & 0.024 & & & 49 & NGC 5018 (disturbed) \\
\hline $1322-428$ & 132232 & -424530 & $\mathrm{P}, \mathrm{BE}, \mathrm{W}$ & $\begin{array}{r}7.9 \\
12.3\end{array}$ & $\begin{array}{r}323 \\
2083\end{array}$ & $e m+a b$ & 1330 & $1,21,36$ & $\begin{array}{l}3,4,5 \\
3,4,5\end{array}$ & 21,60 & NGC 5128; Centaurus A \\
\hline $\begin{array}{l}1339-479 \\
1341-269\end{array}$ & $\begin{array}{l}133956 \\
1341 \quad 15\end{array}$ & $\begin{array}{l}-475449 \\
-265848\end{array}$ & $\begin{array}{l}\mathrm{P}, \mathrm{W} \\
\mathrm{P}\end{array}$ & & $\begin{array}{l}2983 \\
6739\end{array}$ & & $<0.05$ & $\begin{array}{l}1,47,56 \\
1,2,38\end{array}$ & & $\begin{array}{l}1,14,56 \\
12,38\end{array}$ & IC 4320 (misident: \\
\hline $\begin{array}{l}1350+317 \\
1351+405\end{array}$ & 003 & 314134 & $S$ & $\begin{array}{r}15.0 \\
1.5\end{array}$ & $\begin{array}{r}13558 \\
3096\end{array}$ & & $\begin{array}{r}4.55 \\
<0.10\end{array}$ & $22,45,57,59$ & 3,4 & 45 & $\begin{array}{l}\text { IC } 4370 \text { in ref. } 1 \text { ) } \\
\text { UGC 08782; } 36293\end{array}$ \\
\hline $\begin{array}{l}1351+405 \\
1352-336\end{array}$ & 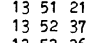 & $\begin{array}{rl}40 & 3232 \\
-33 & 3925\end{array}$ & $\begin{array}{l}\text { D } \\
\text { I }\end{array}$ & 12.5 & 3096 & & $<0.10$ & & & & NGC 5354 \\
\hline $\begin{array}{l}1353+055 \\
1405+552\end{array}$ & 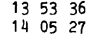 & $\begin{array}{l}052958 \\
551428\end{array}$ & ${ }_{P}^{M, D}$ & $\begin{array}{l}11.2 \\
12.4\end{array}$ & $\begin{array}{l}1081 \\
2136\end{array}$ & $e m+a b$ & 0.25 & $1,23,24,32$ & $\begin{array}{l}4,9,11 \\
5,11\end{array}$ & 7,23, & NGC 5363 \\
\hline $1426-295$ & 142651 & -293134 & $\mathrm{I}(03)$ & & 6845 & & & $\begin{array}{l}1,2 \\
1,2,7\end{array}$ & $\begin{array}{l}5,11 \\
5,11\end{array}$ & 7 & ${ }_{\text {NGC }}^{N G C-P C}$ \\
\hline $\begin{array}{l}\begin{array}{l}1442-137 \\
1444-304\end{array} \\
140\end{array}$ & 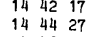 & $\begin{array}{lll}-13 & 44 & 05 \\
-30 & 26 & 09\end{array}$ & $\stackrel{0}{s}$ & & & & 0.017 & 1,51 & $3,7,11$ & : & NGC 5745 (see Table 2) \\
\hline $\begin{array}{l}\begin{array}{l}1448+635 \\
1459-724\end{array} \\
\end{array}$ & $\begin{array}{l}144809 \\
145929\end{array}$ & $\begin{aligned} 632830 \\
-722512\end{aligned}$ & $\begin{array}{c}2(+03) \\
s\end{array}$ & 14.3 & 12564 & & 3.71 & $4,25,57$ & 11 & 61 & IC $1065 ; 3 C 305=4 C 63.21$ \\
\hline $1500-722$ & $\begin{array}{lll}15 & 0027 & 27\end{array}$ & $\begin{array}{ll} & \end{array}$ & & 13.5 & 2933 & & $<0.040$ & 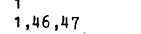 & & & NGC 5 \\
\hline $\begin{array}{l}15322+237 \\
1637+826\end{array}$ & $\begin{array}{ll}15532 & 48 \\
16 & 3757\end{array}$ & $\begin{array}{l}2340 \\
823818\end{array}$ & $\stackrel{I}{P(S ?)}$ & 14.4 & $\begin{array}{l}5558 \\
7131\end{array}$ & $\mathrm{em}$ & $\begin{array}{l}0.42 \\
2.3\end{array}$ & & 11. & $40, "$ & d) \\
\hline $1934-638$ & 193449 & $\begin{array}{l}-6634912 \\
-6312\end{array}$ & $P$ & & $\begin{array}{r}74481 \\
5481\end{array}$ & & ${ }_{16.3}^{2.3}$ & $\begin{array}{l}26,33,57 \\
27,34,35,57\end{array}$ & 6,11 & 27 & $\begin{array}{l}\text { NGCC } 6251 \\
\text { PKS } 1934-\end{array}$ \\
\hline $\begin{array}{l}1957+405 \\
2048-573\end{array}$ & $\begin{array}{l}195744 \\
204812\end{array}$ & $\begin{array}{rl}40 & 3545 \\
-57 & 1530\end{array}$ & $\begin{array}{l}\mathrm{P}(?) \\
0\end{array}$ & & 3405 & & 1495 & 28,57 & $3,4,5$ & 28 & Cy grus $\mathrm{A}=3 \mathrm{C} 405$ \\
\hline $2048-573$ & & & 0 & 13.0 & 3405 & $\mathrm{em}$ & 1.95 & $2,29,30,41$ & & 29,30 & $\begin{array}{l}\text { IC 5063; PRS 2048-57 (radio } \\
\text { ID uncertain) }\end{array}$ \\
\hline $\begin{array}{l}2105-365 \\
2116+262\end{array}$ & $\begin{array}{llll}21 & 05 & 12 \\
21 & 16 & 06\end{array}$ & $\begin{aligned}-363540 \\
26 \\
1406\end{aligned}$ & $\begin{array}{l}P \\
0\end{array}$ & & 5128 & nd & $\begin{array}{l}0.007 \\
0.018\end{array}$ & $4^{1,5}$ & & & NGC \\
\hline $\begin{array}{l}2128-430 \\
2137-428\end{array}$ & $\begin{array}{l}212834 \\
213706\end{array}$ & $\begin{array}{ll}-43 & 0348 \\
-42 & 46\end{array}$ & s & 13.4 & 240 & & 0.0032 & 1,2 & & 7 & ${ }_{A A}, 2116+26$ \\
\hline $2206-474$ & $\begin{array}{lll}22 & 06 & 12\end{array}$ & $-47 \quad 2500$ & 0 & $\begin{array}{l}12.4 \\
11.4\end{array}$ & 1737 & & 0.30 & $\begin{array}{l}46,47 \\
2,48,62\end{array}$ & & 62 & NGC 7213 ( \\
\hline $\begin{array}{l}2210-264 \\
2229+391\end{array}$ & 221019 & $\begin{array}{lll}-26 & 23 & 42\end{array}$ & 0 & & & & & & & & NGC \\
\hline $\begin{array}{l}22299+39191 \\
2255+129\end{array}$ & 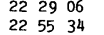 & $\begin{array}{lll}39 & 06 & 11 \\
12 & 51 & 58\end{array}$ & $\begin{array}{l}0 \\
p\end{array}$ & 14.3 & 5413 & & $\begin{array}{l}3.21 \\
0.054\end{array}$ & & 6 & & \\
\hline $2317+169$ & & $\begin{array}{l}165712 \\
16\end{array}$ & $\mathrm{D}, \mathrm{P}$ & 12.8 & 1864 & em & 0.054 & $1,2,31$ & & 40 & $\begin{array}{l}\text { NGC } 7432 \\
\text { NGC } 7625 \text { (m) }\end{array}$ \\
\hline $\begin{array}{l}2323-400 \\
2336+157\end{array}$ & $\begin{array}{lll}23 & 23 & 31 \\
23 & 36 & 14\end{array}$ & $\begin{array}{rll}-40 & 02 & 47 \\
15 & 41\end{array}$ & $\begin{array}{l}0 \\
0\end{array}$ & & & & 0.029 & 1,5 & & & \\
\hline $\begin{array}{l}23360+157 \\
2350-297\end{array}$ & & & I & & & & & & & & NGC 7722 \\
\hline $\begin{array}{l}2353-301 \\
2357-287\end{array}$ & $\begin{array}{l}235313 \\
23 \\
23\end{array} 5757$ & 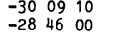 & $\begin{array}{l}0 \\
I\end{array}$ & & & & & 1 & & & \\
\hline
\end{tabular}


TABLE II. Galaxies which may be related to the dusty ellipticals.

\begin{tabular}{|c|c|c|c|c|c|c|c|c|c|c|c|}
\hline $\begin{array}{l}\text { (1) } \\
\text { ID }\end{array}$ & $\begin{array}{l}\text { (2) } \\
\mathrm{RA}(1950)\end{array}$ & $\begin{array}{l}(3) \\
\operatorname{DEC}(1950)\end{array}$ & $\begin{array}{l}\text { (4) } \\
\text { TYPE }\end{array}$ & ${ }_{B}^{(5)}$ & $\begin{array}{l}(6) \\
v\end{array}$ & $\begin{array}{l}\text { (7) } \\
\mathrm{HI}\end{array}$ & $\begin{array}{l}(8) \\
\mathrm{S}(1.4 \mathrm{GHz})\end{array}$ & $\begin{array}{l}\text { (9) } \\
\text { REFERENCES }\end{array}$ & $\begin{array}{l}10) \\
\text { NOTES }\end{array}$ & $\begin{array}{l}i 11) \\
\text { PHOTO }\end{array}$ & $\begin{array}{l}\text { (12) } \\
\text { COMMENTS }\end{array}$ \\
\hline $\begin{array}{l}0122+035 \\
0610+784 \\
1225+133 \\
1442-137\end{array}$ & $\begin{array}{lll}01 & 22 & 00 \\
06 & 10 & 45 \\
12 & 25 & 13 \\
14 & 42 & 17\end{array}$ & $\begin{array}{rrr}03 & 31 & 54 \\
78 & 2230 \\
13 & 1706 \\
-13 & 44 & 05\end{array}$ & $\begin{array}{l}0 \\
0 \\
0 \\
0\end{array}$ & $\begin{array}{l}12.05 \\
11.2 \\
11.9\end{array}$ & $\begin{array}{r}2272 \\
1028 \\
182\end{array}$ & $\begin{array}{l}\mathrm{em} \\
\mathrm{em} \\
\mathrm{em}\end{array}$ & $\begin{array}{l}0.25 \\
1.20 \\
0.30 \\
0.017\end{array}$ & $\begin{array}{l}13 \\
13,66,67 \\
13 \\
51\end{array}$ & $\begin{array}{l}2,3,4 \\
2,3,4 \\
2,3,4 \\
2,3,7\end{array}$ & $\begin{array}{l}40 \\
40 \\
40\end{array}$ & $\begin{array}{l}\text { NGC } 520 \\
\text { NGC } 2146 ; 4 C 78.06 \\
\text { NGC } 4438 \\
\text { NGC } 5745\end{array}$ \\
\hline
\end{tabular}

NOTES FOR TABLES 1 AND 2

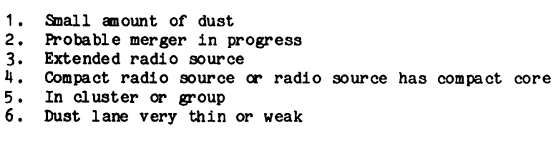
7. Observed by Balick at CrIo, 1983
8. Dwarf elliptical
9. Has close or 1nteracting companion
11. Observed by Ebneter and Balick at KPNO, 1983 May

REFERENCES FOR TABLES 1 AND 2

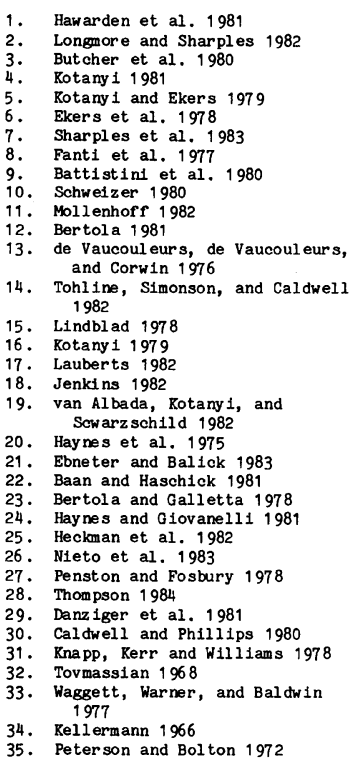

purely optical reasons than in the northern hemisphere sample, because it is easier to identify the dust lanes on the southern sky survey than on the Palomar Sky Survey. This effect was discussed by Hawarden et al. (1981). Galaxies with diffuse, irregular dust are very difficult to identify on either sky survey. Small dusty galaxies like NGC 185, NGC 205, and, possibly, NGC 404, are impossible to identify as dusty systems at even moderate distances and are therefore certain to be severely under-represented. Ellipticals like NGC 315 and NGC 1052 which contain only small amounts of dust will also be poorly represented, as this type of dust is usually discovered only when the nuclear regions of a galaxy are carefully scrutinized. There may be a bias against galaxies with dust lanes along the optical major axis; they are difficult to distinguish from early-type spirals. (The difference between a dusty elliptical and normal SO or Sa galaxy frequently becomes a matter of opinion which can not properly be decided by consideration of bulge flatness.) Finally, there may be a bias towards radio galaxies, particularly in the case of galaxies with small amounts of dust, as such galaxies are more likely to have been subjected to careful study.

The dusty ellipticals present a problem for conventional classification schemes because they do not fit easily into

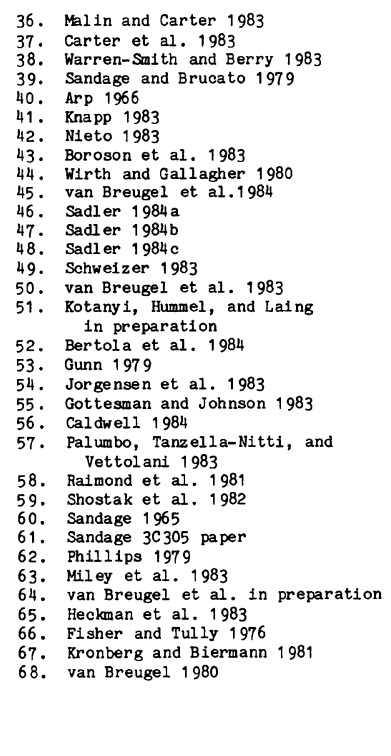

them. These galaxies are most commonly classified as I 0p and S0, with a few described as Sap (e.g., NGC 7625). However, as noted by Kotanyi (1981), they are better described as ellipticals with dust. It would seem, therefore, that some means of organizing and classifying dusty ellipticals would be useful. Hawarden et al. (1981) proposed such a scheme, and it has been adopted here in a somewhat modified form. In particular, two new classes have been added and one has been deleted. The system used in this paper, therefore, is as follows:

$\mathrm{O}=$ oblate-dust lane lies parallel to the optical major axis of the galaxy (this class may be contaminated by normal S0 or Sa galaxies)

$\mathbf{P}=$ prolate - dust lane lies parallel to the optical minor axis of the galaxy

$\mathrm{S}=$ skew-dust lane does not lie parallel to either optical axis of the galaxy

$\mathrm{I}=$ indeterminate-stellar component of galaxy appears spherical; dust is in a well-defined lane

$\mathrm{D}=$ diffuse - dust is distributed in irregular patches in the galaxy

$\mathbf{M}=$ multiple - galaxy has two dust lanes, one on the optical major axis, one on the optical minor axis 
(The terms "oblate" and "prolate" are purely mnemonic and are not meant to imply anything about the true figure of the galaxy.) These classes could be profitably appended to the conventional classifications; for instance, NGC 5128 (Centaurus A) could be described as an E1 dP, where the " $d$ " would be for dust, with the dust morphology afterwards.

Table I is the catalog. All galaxies are identified on the IAU system, with NGC, IC, and other identifications given in column 13, "comments." The "type,"or morphological classification, is given in column 4; these are taken from the literature in most cases, as verified by inspection on the sky surveys. The classes are given as above, with three additional descriptive symbols:

$\mathrm{W}=$ warped-dust lane is warped

$\mathrm{BE}=$ bright edge-dust lane has a bright edge, possibly due to a stellar disk

$\mathrm{U}=$ unknown-dust type could not be determined but galaxy has been identified as containing dust

These symbols are intended to convey the appearance of the galaxy. Column 7 indicates whether or not neutral hydrogen has been observed in the galaxy: "em" = H I emission detected; " $a b "=\mathrm{H}$ I absorption detected; "em $+\mathrm{ab}$ " = both emission and absorption detected; and "nd" = not detected. A reference is given in column 10 where appropriate. Column 8 gives the radio flux (in Jy) at $1.4 \mathrm{GHz}$. If a flux at 1.4 $\mathrm{GHz}$ was not available, the flux was estimated from the published flux (usually at $5 \mathrm{GHz}$ ) using a spectral index of -0.7 . An asterisk $\left({ }^{*}\right)$ in column 12 means that a photograph of the galaxy appears in this paper (see Sec. III).

Table II lists four galaxies which may be related to the dusty ellipticals. One of these, NGC 5745 (see Fig. 1 and Fig. 2 [Plate 14]), is included in both tables. All of these galaxies show evidence of a recent interaction, but all have dust lanes which are remarkably settled in appearance. All these galaxies are radio sources, and the three which have been investigated all contain neutral hydrogen. (This list is by no means exhaustive; the galaxies were chosen on the basis of their resemblance to NGC 5745, which appears to be a normal dusty elliptical on short exposures.) We note that none of the various $\mathrm{H}$ I velocities measured for NGC 4438 are in accord with its otherwise almost certain membership in the Virgo cluster.

\section{OBSERVATIONS OF DUST LANES}

The literature is replete with overexposed photographs of early-type galaxies in which evidence of dust absorption is impossible to discern. An optimum strategy for the detection of the dust would be far-infrared imaging, since the dust can be seen in emission against a dark background. The requisite instrumentation is not yet available. Still, the question of whether small or faint dust lanes are common, or even ubiquitous, in early-type galaxies is a haunting one which might bear directly on questions of the figures of ellipticals systems and the source of "fuel" for galactic activity.

In view of the limited linearity of photographic detectors, we have attempted to investigate the occurrence and distribution of dust in a variety of early-type systems using an optical detector with very large dynamic range. The sample includes many objects from Table I visible in the late spring, as well as ellipticals of comparable luminosity in which dust lanes have not been reported previously. The hope is both to detect dust lanes and to investigate their geometry and relation to the nucleus. To this end, a CCD detector was used with the Cassegrain camera on the \#1 0.9-m telescope at Kitt Peak National Observatory in 1983 May. The sky was photometric, and the seeing better than 2 " throughout the run; indeed, the image quality is limited primarily by the guiding of the telescope and the alignment of its optical elements. Repeated exposures were obtained so that the full dynamic range of the CCD detector could be utilized while obtaining reasonable signal-to-noise levels in regions of low surface brightness in the presence of moonlight. Typically two to four 5-min exposures were obtained in each of standard $B$ and $R$ filters for each galaxy. The ratios of nuclear count rates to sky background to rms pixel-to-pixel noise levels are 1000:10:1, although this varies from galaxy to galaxy. At the $f / 7.5$ Cassegrain focus, one pixel corresponds to $0^{\prime \prime} .85$, and a typical stellar image has a FWHM of 2-3 pixels. Each frame was convolved with a circular Gaussian with a FWHM of 3 pixels in order to reduce the effect of random noise still further.

Because of the (uncalibrated) variations of detector response as a function of wavelength within the filter bands, the present data are not on any standard photometric system. Nevertheless, a standard star was observed so that changes in $B-R$ could, in principle, be mapped across each galaxy. The $B$ and $R$ frames were spatially aligned using a two-dimensional cross-correlation program (written by Robert Braun), and then divided by one another (after subtracting the average sky level from the entire frame). $R$ and $B-R$ frames of selected galaxies are shown in Figs. 1(a)-(h). At low isophotal levels, gradients in the sky brightness, resulting from moonlight scattered in the telescope, affect the color distributions. The effect of sky gradients is not subtle and can be clearly seen in the figures.

Variations in $B-R$ can occur because of reddening from the dust, from intrinsic color variations in the stars (i.e., stellar abundance gradients), or from line emission in one or both of the filter passbands; e.g., $\mathrm{H} \alpha$ ). Shown in Fig. 1 are cases in which increases in $B-R$ are correlated with local absorption regions seen in both the $B$ and $R$ frames. This selects in favor of narrow, well-delineated dust features (indeed, every galaxy we found with such red absorption lanes was already known from earlier photographs to be dusty). Thus, the figures primarily help to clarify the geometry of the dust distribution. Further data processing is required in order to detect dust lanes in galaxies in which dust features have not hitherto been seen. (The results of this processing will be published in due course.) However, with the exception of NGC 6251 and a galaxy with a very small angular diameter (in which the details were obliterated by the limited spatial resolution of the images), the $B-R$ images confirm the presence of dust lanes already known to exist.

A brief discussion of individual galaxies and the geometry of their dust distributions follows.

$N G C$ 3528. The dust appears in a disk lying along the major axis of the galaxy. The near side of the disk lies NW of the nucleus. The disk seems to have about the same extent as the galaxy itself. No starlight appears to be coincident with the disk. Whether the galaxy is a true elliptical is problematical, especially since the spheroidal component of the galaxy appears to be highly flattened [see Fig. 1(a)].

NGC 3665. Much like NGC 3528, except that the dusk disk is much smaller than the galaxy. Conceivably, the dust lies in a small ring centered on the nucleus. A similar situation occurs in the radio galaxy NGC $383=3 \mathrm{C} 31$; NGC 3665 is also a radio galaxy [see Fig. 1(b)]. Note that only because of its compact dust configuration NGC 3665 is more likely to be classified as an elliptical system than NGC 3528 is. 


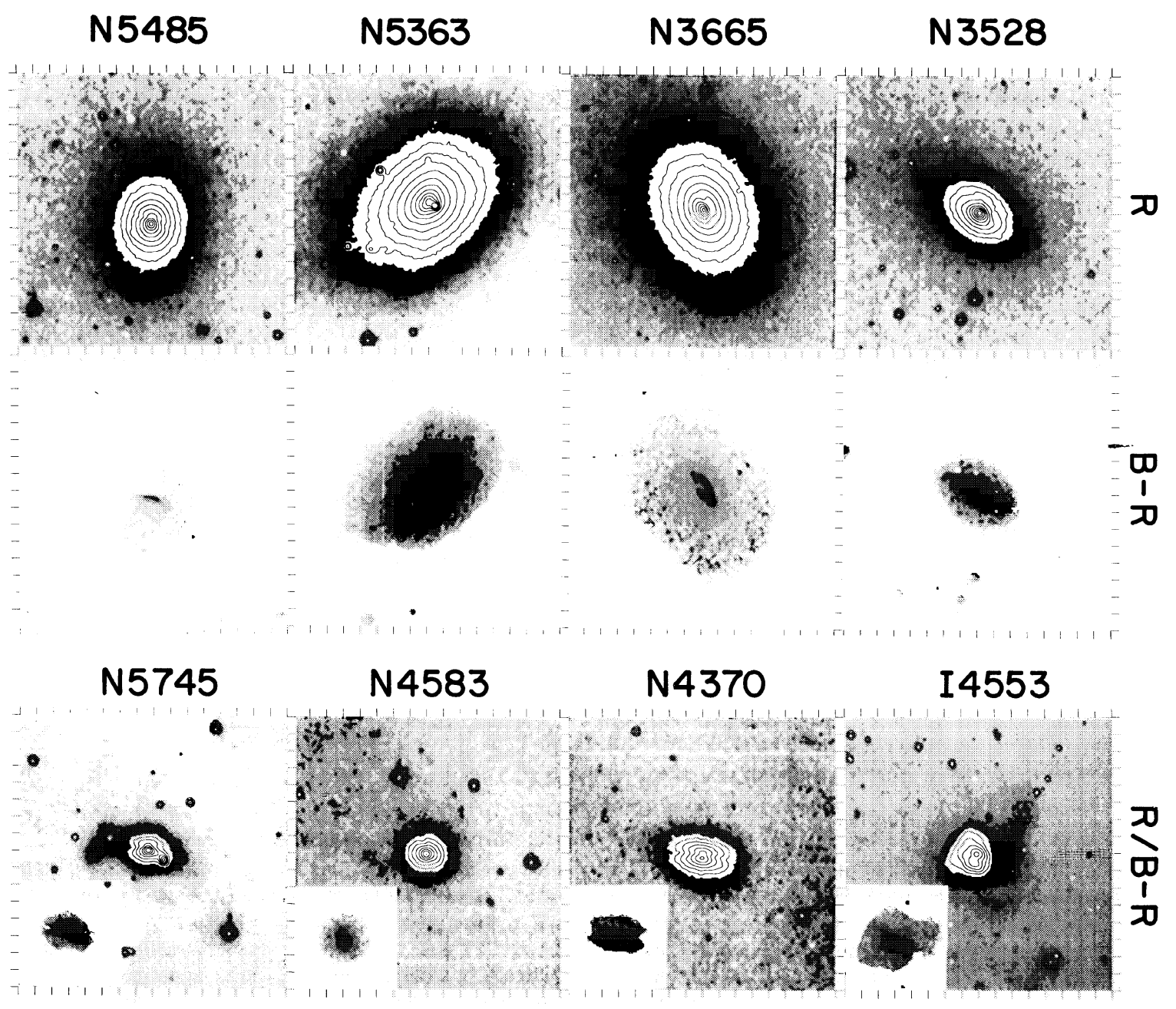

FIG. 1. Distribution of surface brightness observed through an $R$ filter (left-hand panels) and variations in $B-R$ (right-hand panels) for selected dusty elliptical galaxies. For the $R$ frames $(256 \times 256$ pixels), grey levels are shown at lower light levels and contours at higher levels. Contours are drawn every half magnitude (logarithmic spacing), whereas grey levels change in linear steps. Absolute brightness levels have not been calibrated. The $B-R$ variations are indicated by greys (dark implies red colors) with intervals between grey levels in equal fractions of a magnitude. The pixel spacing is $0 " .862$, and tic marks are drawn every 16 pixels $\left(13^{\prime \prime} .8\right)$. Stars have a FWHM of 2".6 (after smoothing). 
$N G C$ 4370. Similar, again, to 3528, except that the dust lane projects almost directly onto the nucleus [Fig. 1(c)].

IC 4553. This galaxy [Fig. 1(h)] appears at first sight to be a double-nucleus galaxy with long tidal tails. If this is true, then the dust lane lies in projection midway between the nuclei. However, the prominence of the dust lane may imply that there is only a single nucleus obscured by the same lane. Infrared observations can clarify this situation. The possibility that the red lane results from $\mathrm{H} \alpha$ emission can not be discounted.

$N G C 4583$. The dust lane lies along the minor axis of the galaxy. Note that if the dust lane were removed the galaxy would appear almost spherical, so the classification of the system as type $\mathbf{P}$ is somewhat problematical [Fig. 1(d)].

$N G C 5363$. The more prominent dust lane lies SW of the nucleus near the major axis. A small second dust lane extends from the nucleus SW to the larger lane [Fig. 1(e)].

$N G C 5485$. The geometry of the dust lane is that of a very thin, highly inclined disk passing close to the nucleus in projection [Fig. 1(f)]. The dust lane lies perpendicular to the galaxy's major axis. Were the dust lane only slightly more inclined, its presence would be extremely difficult to detect. Cases like this argue that dust lanes may be far more common than previously suspected. Note that there is an overall radial color gradient which, we presume, is intrinsic to the stellar component.

NGC 5745. The system shows a striking similarity to NGC 2146, except that NGC 5745 is considerably more distant. A deep CCD image obtained at the 4-m telescope at Cerro Tololo is shown in Fig. 2. Both bright regions in the inner part of the galaxy should be considered part of the highly distorted sytem. The dust lane lies along the longest dimension of this irregularly shaped system. A loop of lowsurface-brightness emission extends in projection from the central part of NGC 5745 to a bright foreground star $90^{\prime \prime}$ to the SW.

\section{PROPERTIES OF DUSTY ELLIPTICALS}

a) Distribution of Morphological Types

While the present catalog is by no means complete (see above), it does provide a reasonably large sample for discussing the general properties of dusty ellipticals. Of the 94 galaxies with known morphological types, $33(35 \%)$ are type $O$, and $25(27 \%)$ are type P. Only $13(14 \%)$ are type D; however, this type may be seriously under-represented in the catalog, as discussed above. Another 13 type S, a small but by no means insignificant fraction of the catalog (two others, NGC 4374 and NGC 708, here classified as P, may in fact be type S; see Kotanyi 1981). These systems are particularly interesting (see below), but very poorly studied. The most unusual type is type M, with only two representatives, NGC 3801 and NGC 5363. Both galaxies have a small dust lane along the minor axis near the nucleus and a larger dust lane along the major axis in the outer parts of the galaxy. Other galaxies are known with orthogonal disks of gas but not dust (NGC 2685, for example, has orthogonal disks of H I: Shane 1980).

The statistics given above differ somewhat from those of Hawarden et al. (1981), who found galaxies of type $O$ to be only slightly more common than those of type P (13 vs 11 , out of a sample of 40). However, they did not include galaxies of type $\mathrm{D}$, and the present list includes a number of galaxies from the list of Longmore and Sharples (1982) which are borderline in the sense that they may include a stellar disk (and would therefore be better classified as early-type spirals). These galaxies were included in this catalog on the basis of their appearance on the sky surveys (and, in the case of NGC 2907, on the basis of a published photograph: Sandage and Brucato 1979), in the absence of any kinematic or photometric evidence for a stellar disk.

\section{b) Radio Properties of Dusty Ellipticals}

Astronomical folklore has it that ellipticals with dust are more likely to be active than are "normal" ellipticals. The archetypal dusty elliptical is NGC 5128, which is the wellknown radio source Centaurus $A$, and the powerful radio galaxies Cygnus A and PKS 1934 - 63 are also dusty ellipticals. [A recent CCD study of Cygnus A by Thompson (1984) demonstrates fairly convincingly that the commonly drawn analogy between Cyg $\mathbf{A}$ and Cen $\mathbf{A}$ is probably incorrect, in the sense that the peculiar double appearance of Cyg $A$ is probably real and not an optical effect caused by the presence of a lane of dust like that in Cen A. However, his images also indicate that, nevertheless, Cyg A probably does contain dust in patches near the nucleus, possibly in a thin lane. None of this disqualifies Cyg A from inclusion in this catalog.] A fairly large fraction (32\%) of the galaxies in Table I have radio fluxes of $30 \mathrm{mJy}$ or more, which is somewhat unusual for a sample this size; Disney and Wall (1977), in a survey of southern E and S0 galaxies at $5 \mathrm{GHz}$, detected 39 of 181 galaxies, or $22 \%$, with a detection limit of $12 \mathrm{mJy}(12$ $\mathrm{mJy}$ at $5 \mathrm{GHz}$ is roughly comparable to $30 \mathrm{mJy}$ at $1.4 \mathrm{GHz}$ ). This result should be treated with caution, however, as the selection effects in the present catalog may be rather insidious.

Other types of activity (e.g., x-ray emission, nuclear emission lines, etc.) in dusty ellipticals have not been systematically studied.

\section{c) Radio Source Orientation}

Kotani and Ekers (1979) and Kotani (1981) have noted a tendency for the radio sources in dusty ellipticals to be oriented roughly perpendicular to the dust lane. None of the galaxies in the present catalog alter this result significantly, although two more galaxies with extended radio sources may be added to the list, NGC 5745 and 1029 - 459; both have relatively weak radio sources which are oriented approximately perpendicular to the respective dust lanes (Kotani, private communication). There are, however, two points worthy of comment. First, two galaxies, IC 5063 and NGC 6251, have been offered as counterexamples to the above suggestion (Danziger et al. 1981; Nieto et al. 1983); both galaxies have radio sources that are parallel to their dust lanes. However, it is not certain that the weak western "lobe" of the radio source PKS 2048-57 is really associated with IC 5063 (if it is, the radio source would be single lobed and highly asymmetric), and the dust lane in NGC 6251 has not yet been confirmed. Further study of these two examples is therefore needed. Second, in two well-studied galaxies of type S, IC 1065 (3C305) and 3C293 [see Heckman et al. (1982) and van Breugel et al. (1984), respectively], and one type-M galaxy, NGC 3801 (van Breugel et al., in preparation), the radio sources are distorted and rather small; they resemble the extended radio sources found in some Seyfert galaxies, particularly NGC 1068 (Wilson and Willis 1980; see also Wilson 1982). It has been suggested that these sources are distorted by the interaction of the radio plasma 
with the gas in the disk, but it is not clear why the radio source in these galaxies should emerge into the disk rather than perpendicular to it, nor why these sources should suffer this type of distortion while that in NGC 6251 apparently does not. None of the remaining S-type galaxies in the cata$\log$ are strong radio sources, with the possible exceptions of NGC 4374 and NGC 708, in which the dust lanes are, however, only weakly skewed. (Both radio sources in these galaxies are almost perpendicular to their respective dust lanes.)

\section{d) Neutral Hydrogen}

Fourteen of the one hundred galaxies in Table I have neutral hydrogen in either emission or absorption, occasionally both; upper limits on $21 \mathrm{-cm}$ emission are available for six others. The others have not been studied. The neutral-hydrogen content is in itself not terribly surprising - where one finds dust, one ought to also find gas-but the quantitative results are interesting. NGC 5128 has been well studied and has been found to contain about $10^{9} \mathrm{M}_{\odot}$ of $\mathrm{H} \mathrm{I}$ (Gardner and Whiteoak 1976), which VLA maps (Shostak et al. 1983) show to be in the same configuration as the dust. However, NGC 1316 (Fornax A) contains very little $\mathrm{H} \mathrm{I}$, despite its superficial resemblance to NGC 5128 (Jenkins 1983) [although it does contain a disk of ionized gas (Schweizer 1980)]; and M84 also contains very little, if any, neutral hydrogen (Heckman et al. 1983). There is no reason to assume that the gas must be neutral, of course, so studies of the ionized and/or molecular gas content of the sample would be very interesting. [Caldwell $(1984(b)]$ has recently looked for [O II] emission in a sample of about one hundred elliptical galaxies, including some 15 dusty ellipticals. He detects almost all of the dusty galaxies.] It is also interesting to note that there does not seem to be any preference for radio galaxies in the sample to be the ones which contain neutral hydrogen, except that very few of the radio-quiet galaxies have been searched.

\section{e) Kinematics and Dynamics}

The kinematics of a small number of these galaxies have been studied by various authors; they may provide important clues to both the origin of the dust and gas and the intrinsic figure of the underlying elliptical galaxy. Galaxies with well-studied kinematics are NGC 5128 (Graham 1979); NGC 1316 (Schweizer 1980); NGC 1947 (Mollenhoff 1982); NGC 5626, NGC 5363, NGC 7070A, and the anonymous galaxy 0151-498 (Sharples et al. 1983); NGC 3108 and NGC 5266 (Caldwell 1984a); NGC 612 (Goss et al. 1980); and IC 5063 (Caldwell and Phillips 1981). In all cases, the dust and associated gas appear to be in a disk configuration which is rotating, as would be expected. The stellar kinematics vary from galaxy to galaxy. In NGC 1947 (Mollenhoff 1982), NGC 5128 (Graham 1979), and 0151 - 498 (Sharples et al. 1983), all of which are type-P galaxies, the gas disks are found to rotate about the optical major axis of the galaxy, while the stellar component rotates very slowly, if at all. There is no evidence in NGC 1947 for rotation of the stellar component about either axis; in NGC 5128 there is weak (and unverified) evidence for rotation of the stellar component about the minor axis, i.e., perpendicular to the rotation of the dust lane (Danziger et al., quoted in Lake and Normal 1983). Such a situation is known to occur in NGC 5266 (Caldwell 1984a) and in NGC 1316 (Schweizer 1980), where the measurements are based on ionized gas not directly asso- ciated with the dust, which has a more irregular distribution. The same thing occurs in a number of southern dusty ellipticals of type $\mathbf{P}$ which have recently been studied (Mollenhoff, private communication). Caldwell (1984a) concluded from his measurements of NGC 5266 that the galaxy must be triaxial, a conclusion which probably also applies to the other cases where the dust and stars have very different rotations. Another case which is extremely interesting is that of NGC 5363, the only one of the type-M galaxies which has been studied kinematically. In NGC 5363, Sharples et al. (1983) found that the gas rotates about the major axis near the nucleus, and about the minor axis in the outer regions of the galaxy; the stellar component rotates about the minor axis. NGC 7070A, the only type-S galaxy to have been studied, shows no stellar rotation (Sharples et al. 1983). In IC 5063 (Caldwell and Phillips 1981), NGC 612 (Goss et al. 1980), and NGC 5626 (Sharples et al. 1983), the gas and stars co-rotate around the minor axis (these are all type-O systems). Two of these systems show some evidence for the presence of a stellar disk.

\section{f) Relationship to Other Types of Peculiar Galaxies}

The dusty elliptical galaxies may be related to several other types of peculiar systems, specifically the M82-type irregular of "amorphous" galaxies, elliptical galaxies_with large gas content (but not apparent dust), and the so-called S0 galaxies with polar rings. The hypotheses which have been proposed to explain all three types of galaxies are very similar.

The so-called Irr II galaxies form a rather heterogeneous class in the sense that the class encompasses a variety of morphological types; a number of the galaxies in the present catalog have previously been classified as Irr II. There are a fair number of Irr IIs which resemble the well-known galaxy M82; these were described by Sandage and Brucato (1979) as amorphous. These systems tend to be highly flattened (at least those examples that are seen edge-on) and contain large amounts of dust in irregular patterns; however, they show no evidence for spiral structure. Some examples contain gaseous filaments (Krienke and Hodge 1974). Many such galaxies are found in close proximity to large spiral galaxies (e.g., M82 and NGC 3077, which are in a group with the spiral M81). This close relationship with spiral galaxies suggests that an interaction with the spiral has resulted in the acquisition of gas by the smaller galaxy. (Something similar may have happened with the dwarf ellipticals NGC 185 and NGC 205.) However, other examples, for instance NGC 5253, are more or less isolated. Most of the amorphous galaxies seem to have recently undergone a burst of star formation, possibly due to the recent infall of gas into a previous gas-free system. The highly irregular distribution of the dust in these systems may imply that the dust and gas are, in fact, newly acquired; since small ellipticals do seem to be rotationally flattened, unlike giant ellipticals (Davies et al. 1983), one would perhaps expect that the gas would settle down into a preferred plane rather quickly.

A small but significant number of ellipticals are known which contain neutral hydrogen (see Knapp 1983 for a review); many of these are also dusty ellipticals, but few are not known to contain any dust (although the possibility of dust being found in a careful search, especially of the nuclear regions, should not be ignored). A number of other ellipticals are known which contain ionized gas (see, e.g., Caldwell 1984b). Since ellipticals are traditionally believed to be gas 
poor as well as dust free, it is obvious that there may be some connection to the dusty ellipticals. Many of these "gassy" ellipticals are active (as are many dusty ellipticals), but this may, of course, be a selection effect due to the tendency to look for gas in active ellipticals as a source of fuel for the nucleus. The number of inactive ellipticals which are gassy is not known, since it is not as easy to search for gas as for dust. The origin of the gas is also a subject of debate; see below.

A number of galaxies have been found which appear to be S0 galaxies with a ring of material (typically stars, gas, and dust) in a polar orbit. The archetype for this class is NGC 2685 , sometimes called the "spindle", although there is considerable evidence that the so-called spindle is in fact a disk (Schechter and Gunn 1978). Shane (1980) found that NGC 2685 contains two disks of $\mathrm{H} \mathrm{I}$ which are oriented more or less perpendicularly to each other. A number of other galaxies of this type have been discovered recently; the best known is NGC 4650A, but all present the same general appearance. Those for which the kinematics have been studied have generally been found to be disk systems, rather than prolate spheroids (Schweizer et al. 1983) as was once believed.

\section{CONCLUSION}

The dusty elliptical galaxies form an interesting class for several reasons: they seem to be more active than non-dusty ellipticals; they provide information about the true figures of elliptical galaxies and may therefore be important to our understanding of all ellipticals; they may be the merger remnants that many authors believe should be found. The number of dusty early-type galaxies is in fact consistent with the number of expected merger remnants; many of the known examples demonstrate characteristics which some authors believe to be indicative of mergers (Schweizer 1983): shells, tails, a generally disturbed appearance. Furthermore, it is difficult to understand the presence of significant amounts of gas and dust in ellipticals without invoking some type of accretion event; in no example of a dusty elliptical are the kinematics consistent with the gas coming from stellar mass loss or ejection from the nucleus; nor can Kotanyi's (1981) idea that active ellipticals can "protect", and therefore re- tain, their gas explain the presence of significant gas/dust disks in nonactive ellipticals. It therefore seems more likely that the (suspected) high rate of activity in dusty ellipticals means that the disk provides a source of fuel for the nucleus, although this of course begs the question of how the "monster" formed.

Dusty ellipticals may also be important to the study of activity in galaxies in another way. It is well known that active spirals rarely, if ever, possess giant double-lobed radio sources of the type that are commonly found in radio ellipticals. One theory to explain this result has been that spirals of course contain a disk which contains a great deal of gas, and the presence of this gas disk may inhibit the formation of a classical double-lobed radio source. However, some dusty ellipticals have substantial disks of gas (e.g., NGC 5128 = Centaurus A), but also have large double-lobed radio sources. This suggests that perhaps the galaxy must acquire a large central engine before it acquires a gas disk, or perhaps there is some qualitative difference between the engines formed by spiral galaxies and the engines formed by ellipticals.

Further study of the dusty ellipticals is clearly necessary. Searches for dust in both active and inactive ellipticals should help to clarify the suspected connection between dust and radio sources. Information is also needed on other types of activity in dusty ellipticals, e.g., $x$-ray sources or nuclear emission-line sources, etc. The search for dust in ellipticals would ideally be carried out in the far infrared, where the orientation of the dust and gas disk is less important than it is in the visual. Further kinematic studies of dusty ellipticals are necessary both to clarify our understanding of the figures of elliptical galaxies and to search for clues to the origin of the gas and dust in these systems.

It is a pleasure to thank the staff at Kitt Peak, especially Jeanette Barnes, for their helpful advice and assistance with the observations and data calibration. Financial support was provided by grants from the National Science Foundation (AST 83-8208041) and the Netherlands Foundation for Pure Research.

\section{REFERENCES}

Arp, H. C. (1966). Atlas of Peculiar Galaxies (California Institute of Technology, Pasadena).

Baan, W. A., and Haschick, A. D. (1981). Astrophys. J. Lett. 243, L143.

Battistini, P., Bonoli, F., Silvestro, S., Fanti, R., Gioia, I. M., and Giovannini, G. (1980). Astron. Astrophys. 185, 101.

Bertola, F. (1981). Sky Telesc. 61, 380.

Bertola, F., and Galetta, G. (1978). Astrophys. J. Lett. 226, L115.

Bertola, F., Bettoni, D., Rusconi, L., and Sedmak, G. (1984). Astron. J. 89, 356.

Boroson, T. A., Thompson, I. B., and Schectman, S. A. (1983). Astron. J. 88, 1707.

Butcher, H. R., van Breugel, W. J. M., and Miley, G. K. (1980). Astrophys. J. 235, 749.

Caldwell, N. (1984a). Astrophys. J. 278, 96.

Caldwell, N. (1984b). Publ. Astron. Soc. Pac. 96, 287.

Caldwell, N., and Phillips, M. M. (1981). Astrophys. J. 244, 447.

Carter, D., Jorden, P. R., Thorne, D. J., Wall, J. V., and Straede, J. C. (1983). Mon. Not. R. Astron. Soc. 205, 377.

Danziger, I. J., Goss, W. M., and Wellington, K. J. (1981). Mon. Not. R. Astron. Soc. 196, 845.

Davies, R. I., Efstathiou, G., Fall, S. M., Illingworth, G., and Schechter, P.
L. (1983). Astrophys. J. 266, 41.

de Vaucouleurs, G., de Vaucouleurs, A., and Corwin, H. G. (1976). Second Reference Catalog of Bright Galaxies (University of Texas, Austin). de Zeeuw, T. (1984). Ph.D. thesis, University of Leiden.

Disney, M. J., and Wall, J. V. (1977). Mon. Not. R. Astron. Soc. 179, 235.

Ebneter, K., and Balick, B. (1983). Publ. Astron. Soc. Pac. 95, 675.

Ekers, R. D., Goss, W. M., Kotanyi, C. G., and Skellern, D. J. (1978). Astron. Astrophys. 69, L21.

Fanti, C., Fanti, R., Gioia, I. M., Lari, C., Darma, P., and Ulrich, M.-H. (1977). Astron. Astrophys. Suppl. 29, 279.

Fisher, J. R., and Tully, R. F. (1976). Astron. Astrophys. 53, 397.

Gardner, F. F., and Whiteoak, J. B. (1976). Proc. Astron. Soc. Aust. 3, 63.

Goss, W. M., Danziger, I. J., Fosbury, R. A. E., and Boksenberg, A. (1980). Mon. Not. R. Astron. Soc. 190, 23P.

Gottesman, S. T., and Johnson, D. W. (1983). In Internal Kinematics and Dynamics of Galaxies, IAU Symposium No. 100, edited by E. Athanassoula (Reidel, Dordrecht), p. 307.

Graham, J. A. (1979). Astrophys. J. 232, 60.

Gunn, J. E. (1979). In Active Galactic Nuclei, edited by C. Hazard and S.

Mitton (Cambridge University, Cambridge, England), p. 213.

Hawarden, T. G., Elson, R. A. W., Longmore, A. J., Tritton, S. B., and 
Corwin, H. G., Jr. (1981). Mon. Not. R. Astron. Soc. 196, 747.

Haynes, M. R., and Giovanelli, R. (1981). Astrophys. J. Lett. 246, L105.

Haynes, R. F., Huchtmeier, W. K. H., Siegman, B. C., and Wright, A. E (1975). A Compendium of Radio Measurements of Bright Galaxies (CSIRO Division of Radiophysics, Sydney).

Heckman, T. M., Miley, G. K., Balick, B., van Breugel, W. J. M., and Butcher, H. R. (1982). Astrophys. J. 262, 529.

Heckman, T. M., Balick, B., van Breugel, W. J. M., and Miley, G. K. (1983). Astron. J. 88, 583.

Jenkins, C. R. (1982). Mon. Not. R. Astron. Soc. 200, 705.

Jenkins, C. R. (1983). Mon. Not. R. Astron. Soc. 205, 1321.

Jorgensen, H. E., Norgaard-Nielsen, H. U., Pedersen, H., Rasmussen, I. L., and Schnopper, H. (1983). Astron. Astrophys. 122, 301.

Kellermann, K. I. (1966). Aust. J. Phys. 19, 195.

Knapp, G. R. (1983). In Internal Kinematics and Dynamics of Galaxies, IAU Symposium No. 100, edited by E. Athanassoula (Reidel, Dordrecht), p. 297.

Knapp, G. R., Kerr, F. J., and Williams, B. A. (1978). Astrophys. J. 222, 800.

Kotanyi, C. G. (1979). Astron. Astrophys. 74, 156.

Kotanyi, C. G. (1981). Ph.D. thesis, University of Leiden.

Kotanyi, C. G., and Ekers, R. D. (1979). Astron. Astrophys. 78, L1.

Krienke, O. K., Jr., and Hodge, P. W. (1974). Astron. J. 79, 1242.

Kronberg, P. P., and Biermann, P. (1981). Astrophys. J. 243, 89.

Lake, G., and Norman, C. (1983). Astrophys. J. 270, 51.

Lauberts, A. (1982). ESO / Uppsala Catalogue of the ESO (B) Sky Survey (European Southern Observatory, Munich).

Lindblad, P. O. (1978). The Messenger 10, 21.

Longmore, A. J., and Sharples, R. M. (1982). Mon. Not. R. Astron. Soc. 201, 111.

Malin, D. F., and Carter, D. (1983). Astrophys. J. 274, 534

Miley, G. K., Norman, C., Silk, J., and Fabbiano, G. (1983). Astron. Astrophys. 122, 330.

Mollenhoff, C. (1982). Astron. Astrophys. 108, 130.

Morgan, W. W. (1958). Publ. Astron. Soc. Pac. 70, 364.

Morgan, W. W. (1959). Publ. Astron. Soc. Pac. 71, 394.

Nieto, J.-L. (1983). In Astrophysical Jets, edited by A. Ferrari and A. G. Pacholczyk (Reidel, Dordrecht), p. 113.

Nieto, J.-L. Coupinot, G., Lelievre, G., and Madsen, C.(1983). Mon. Not. R. Astron. Soc. 203, 39P.

Palumbo, G. G. C., Tanzella-Nitti, G., and Vettolani, G. (1983). Catalogue of Radial Velocities of Galaxies (Gordon and Breach, New York).

Penston, M. V., and Fosbury, R. A. E. (1978). Mon. Not. R. Astron. Soc. $183,479$.

Peterson, B. A., and Bolton, J. G. (1972). Astrophys. J. Lett. 173, L19.
Phillips, M. M. (1979). Astrophys. J. Lett. 227, L121.

Quinn, P. (1984). Astrophys. J. 279, 596.

Raimond, E., Faber, S. M., Gallagher III, J. S., and Knapp, G. R. (1981).

Astrophys. J. 246, 708.

Sadler, E. M. (1984a). Astron. J. 89, 23.

Sadler, E. M. (1984b). Astron. J. 89, 34.

Sadler, E. M. (1984c). Astron. J. 89, 53.

Sandage, A. (1961). The Hubble Atlas of Galaxies (Carnegie Institution of Washington, Washington, D.C.).

Sandage, A. (1966). Astrophys. J. 145, 1.

Sandage, A., and Brucato, R. (1979). Astron. J. 84, 472.

Schechter, P. L., and Gunn, J. E. (1978). Astron. J. 83, 1360.

Schweizer, F. (1980). Astrophys. J. 237, 303.

Schweizer, F. (1983). In Internal Kinematics and Dynamics of Galaxies, IAU Symposium No. 100, edited by E. Athanassoula (Reidel, Dordrecht), p. 319.

Schweizer, F., Whitmore, B. C., and Rubin, V. C. (1983). Astron. J. 88, 909.

Shane, W. W. (1980). Astron. Astrophys. 82, 314.

Sharples, R. M., Carter, D., Hawarden, T. G., and Longmore, A. J. (1983).

Mon. Not. R. Astron. Soc. 202, 37.

Shostak, G. S., van Gorkom, J. H., Ekers, R. D., Sanders, R. H., Goss, W.

M., and Cornwell, T. J. (1983). Astron. Astrophys. 119, L3.

Thompson, L. (1984). Astrophys. J. Lett. 279, L147.

Tohline, J. E., Simonson, G. F., and Caldwell, N. (1982). Astrophys. J. 252, 92.

Toomre, A. (1977). In The Evolution of Galaxies and Stellar Populations, edited by B. Tinsley and R. Larson (Yale University Observatory, New Haven), p. 401.

Tovmassian, H. M. (1968). Aust. J. Phys. 21, 193.

Tubbs, A. D. (1980). Astrophys. J. 241, 969.

van Albada, T. S., Kotanyi, C. G., and Schwarzschild, M. (1982). Mon. Not. R. Astron. Soc. 198, 303.

van Breugel, W. (1980). Ph.D. thesis, University of Leiden.

van Breugel, W., Heckman, T., Bridle, A., Butcher, H., Strom, R., and Balick, B. (1983). Astrophys. J. 275, 61.

van Breugel, W., Heckman, T., Butcher, H., and Miley, G. (1984). Astrophys. J. 277, 82.

Waggett, P. C., Warner, P. J., and Baldwin, J. E. (1977). Mon. Not. R. Astron. Soc. 181, 465.

Warren-Smith, R. F., and Berry, D. S. (1983). Mon. Not. R. Astron. Soc. 205, 889.

Wilson, A. S. (1982). In Extragalactic Radio Sources, IAU Symposium No.

97, edited by D. S. Heeschen and C. M. Wade (Reidel, Dordrecht), p. 179.

Wilson, A. S., and Willis, A. G. (1980). Astrophys. J. 240, 429.

Wirth, A., and Gallagher, J. S. (1980). Astrophys. J. 242, 469. 




Fig. 2. CCD frame of NGC 5745 obtained at the 4-m telescope at Cerro Tololo through a $V$ filter. The data were obtained under conditions of approximately 1.5 and variable atmospheric transparency. Standard dark count and flat-field correction have been applied.

K. Ebneter and B. Balick (see page 186) 